\title{
Texture Segmentation Using Multichannel Gabor Filtering
}

\author{
M. Sivalingamaiah ${ }^{1}$, B. D. Venakramana Reddy ${ }^{2}$ \\ ${ }^{1,2}$ (ECE Department, MITS, Madanapalle, India)
}

\begin{abstract}
Texture is defined as the regular repetition of an element or pattern on a surface. Texture is an important property of surfaces which characterizes the nature of the surface. An important task in image processing and machine vision is the task of segmenting the texture in an image. Texture segmentation is the process of partitioning an image into regions based on their texture. In this project we would like to propose a new approach using multichannel Gabor filters for the segmentation of colour and grayscale multi textured images. The proposed method includes the design of the constituent Gabor filters. Based on these new Gabor filters, we develop new algorithms for the texture segmentation.
\end{abstract}

Keywords - clustering, Gabor filter, Multichannel, segmentation, Texture

\section{Introduction}

Early a lot of work has to be done on Texture segmentation starting from fundamental filter transforms to the multi resolution techniques. Now the texture segmentation schemes are based on Gabor filters. Recently Texture segmentation was implemented by using single channel scheme which is limited to two textures only. In this project we extended the existing single-channel method for texture segmentation to multi-channel method by using multiple Gabor filters. Based on this extension the present work deals with multi channel texture segmentation of gray scale and colour images. Specifically this project deals with multi channel texture segmentation of a colour and gray scale images to find out the different textures present in the composite image. This paper addresses the design of multiple Gabor filters for segmenting multi-textured images.

The objective of this project is segmentation of colour and gray scale multi texture images using multichannel Gabor filtering. Here we design Gabor filter bank which is used to decompose the input image into number of filtered images by using a set of frequencies orientations that cover spatial frequency space and capture texture information as much as possible. Here care has to be taken so that the filters do not overlap to avoid the aliasing. Section 2 deals with the Gabor filters and basics of segmentation. Section 3 introduces the multichannel Gabor filters and the response of Gabor filter. Section 4 discusses the texture segmentation process. Experimental results are shown in section 4 . Section 5 demonstrates the conclusion and future enhancement to this work.

\section{Gabor Filters}

A Gabor filter is a linear filter whose impulse response is defined by a harmonic function multiplied by a Gaussian function. Because of the multiplication-convolution property (Convolution theorem), the Fourier transform of a Gabor filter's impulse response is the convolution of the Fourier transform of the harmonic function and the Fourier transform of the Gaussian function.

A Gabor filter is a linear filter used for edge detection in image processing which is named after Dennis Gabor. Gabor filter frequency and orientation representations are similar to those of human visual system, for texture representation and discrimination it has been found to be remarkably appropriate. A sinusoidal plane wave has been modulating a 2D Gabor filter which is a Gaussian kernel function in the spatial domain. From one parent wavelet all filters can be generated by dilation and rotation, thus the Gabor filters are self-similar.

Texture Segmentation involves partitioning or segmenting an image into various regions of repetitive patterns or textures with accuracy. The objective of this being to group regions with similar textures which might belong to the same object or class of objects. The process is carried out using the filter-bank mode which uses a set of linear image filters operating in parallel to divide or decompose an input image into several output images. These filters are designed such that they simultaneously focus on a particular range of frequencies and on local spatial interactions and gives rise to the concept of joint space / spatial-frequency decomposition.

\section{Multichannel Gabor Filters}

Here is a brief overview of Gabor Elementary Functions (GEF's) and Gabor Filters are given. GEF's were first defined by Gabor and later extended to 2-D by Daugman.

A GEF is given by

$$
\mathrm{h}(\mathrm{x}, \mathrm{y})=\mathrm{g}\left(x^{\prime}, y^{\prime}\right) \exp [\mathrm{p} 2 \pi(U x+V y)]
$$

Where $\left(x^{\prime}, y^{\prime}\right)=(x \cos \theta+y \sin \theta,-x \sin \theta+y \cos \theta)$ represent rotated spatial-domain rectilinear coordinates. Let $(\mathrm{u}, \mathrm{v})$ denote frequency-domain rectilinear coordinates, $(\mathrm{U}, \mathrm{V})$ represents a particular 2-D frequency. The 
complex exponential is a 2-D complex sinusoid at frequency $\mathrm{F}=\sqrt{U^{2}+V^{2}}$ and $\varphi=\tan ^{-1} \frac{V}{U}$ and specifies the orientation of the sinusoid. The function $\mathrm{g}(\mathrm{x}, \mathrm{y})$ is the 2-D Gaussian.

$$
\mathrm{g}(\mathrm{x}, \mathrm{y})=\frac{1}{2 \pi \sigma_{x} \sigma_{y}} \exp \left\{-\frac{1}{2}\left[\frac{x^{2}}{\sigma_{x}^{2}}+\frac{y^{2}}{\sigma_{y}^{2}}\right]\right\}
$$

Where $\sigma_{x}$ and $\sigma_{y}$ characterize the spatial extent and bandwidth of the filter.

The Gaussian's major and minor axis widths are determined by $\sigma_{x}$ and $\sigma_{y}$, it is rotated by an angle $\theta$ with respect to the positive u-axis and it is centered about the frequency $(\mathrm{U}, \mathrm{V})$. Thus, the GEF acts as a band pass filter. In most cases, letting $\sigma_{x}=\sigma_{y}=\sigma$ is a reasonable design choice. If it assumed that $\sigma_{x}=\sigma_{y}=\sigma$, then the parameter $\theta$ is not needed and the equation of GEF simplifies to

$$
\mathrm{h}(\mathrm{x}, \mathrm{y})=\frac{1}{2 \pi \sigma^{2}} \exp \left\{-\frac{\left(x^{2}+y^{2}\right)}{2 \sigma^{2}}\right\} \exp [j 2 \pi(U x+v Y)]
$$

We now define the Gabor Filter $O_{h}$ by

$$
\mathrm{m}(\mathrm{x}, \mathrm{y})=O_{h}(\mathrm{i}(\mathrm{x}, \mathrm{y}))=|\mathrm{i}(\mathrm{x}, \mathrm{y}) \otimes \mathrm{h}(\mathrm{x}, \mathrm{y})|
$$

Where $i$ is the input image and $m$ is the output.

Accurate segmentation only occurs if the parameters defining the Gabor filters are suitably chosen.

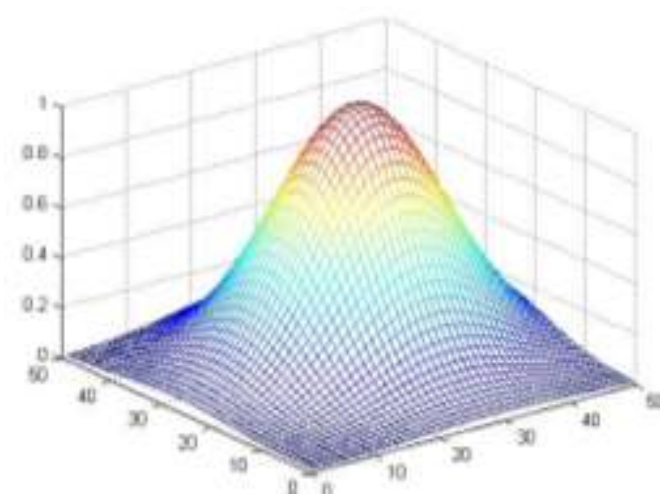

Figure 1 .Multichannel Gabor Filter Responses with parameters (sig1, sig2) $=(10,11),(\mathrm{u}, \mathrm{v})=0.3$

The above diagram shows the responses of Multichannel Gabor Filters for spatial extent in x \&y directions (sig1, sig2 $)=(5,5)$ and frequency-domain rectilinear coordinates $(\mathrm{u}, \mathrm{v})=(0.5,0.5)$. Here we consider the size of the filter mask is $50 \times 50$.

\section{Texture Segmentation}

Original image

Bank of Gabor filters

Filtered images

Feature extraction

Clustering

Segmented image

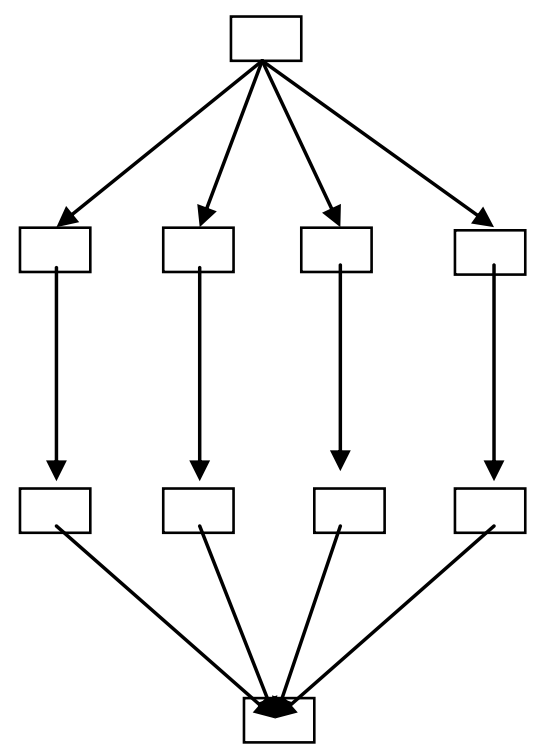

Figure 2.Texture Segmentation 
The process of texture segmentation using multi-channel filtering involves the following steps[12]:

- $\quad$ Filter bank design,

- Decomposition of the input image using the filter bank,

- Feature extraction, and

- $\quad$ Clustering of pixels in the feature space.

\section{(A) Filter Bank Design}

The number of filters required is $\theta \log _{2}\left(N_{c} / 2\right)$

Where as $N_{c}$ the width of an image, $\theta$ is the orientation separation. For an image of width 256 pixels and orientation separation of $30^{\circ}, 42$ filters are required[12].

(B) Filtering Image With Filter Bank

After designing the filter bank, the image was passed through the filter bank then the image was decomposed into the number of textures.

\section{(C) Feature Extraction}

Smoothing can be obtained by applying low pass filtering. In an image the low frequency components represents contrast and intensity, where as the high frequency components represents edges and sharp details present in the image. If we apply smoothing all the low frequency components are allowed and high frequency components are attenuated. That means the contrast $\&$ intensities are highlighted and edges $\&$ sharp details are attenuated. As a result we get the blurred image. However, too much smoothing can have a negative effect on the localization of texture region edges. Image segmentation can be done by detecting the edges. Smoothing filters attenuates the edges. So care has to be taken while applying spatial smoothing[12].

\section{(D) Clustering In The Feature Space}

The final step is to cluster the pixels into a number of clusters representing the texture regions. The algorithm of k-means is as follows:

1. Initialize centroids of K-clusters randomly.

2. Assign each sample to the nearest centroid.

3. Calculate centroids (means) of K-clusters.

4. If centroids are unchanged, done. Otherwise, go to step 2.

When clustering is done, each pixel is labelled with its respective cluster, finally producing the segmented image.

\section{Experimental Results}

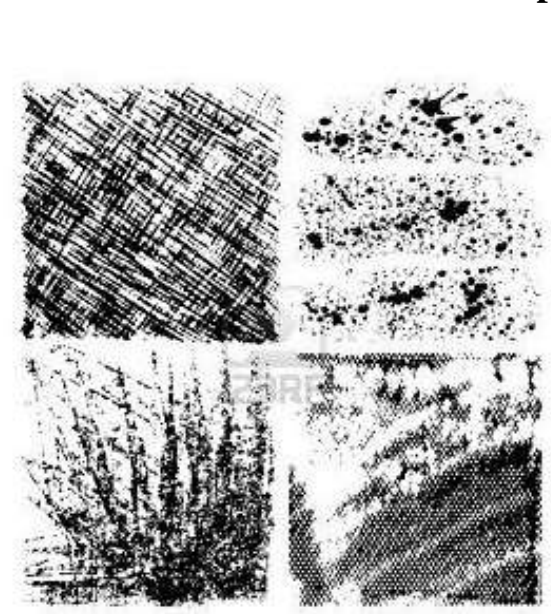

(a)

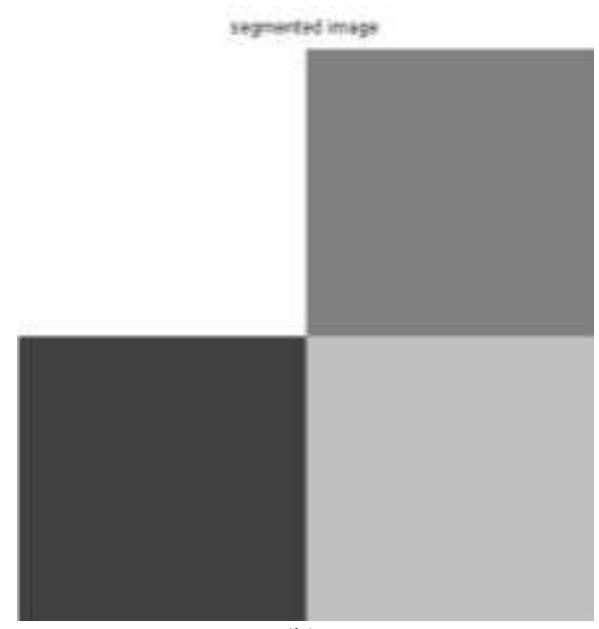

(b)

Figure 3.Segmentation of four texture gray scale image (a) input image (b) segmented image for $\theta=$ $45^{0}$ and $N_{c}=256$ pixels 


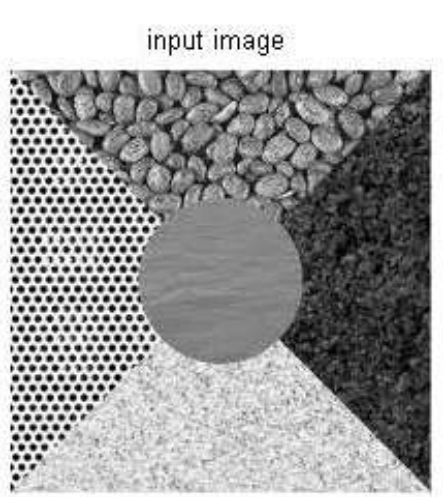

(a)

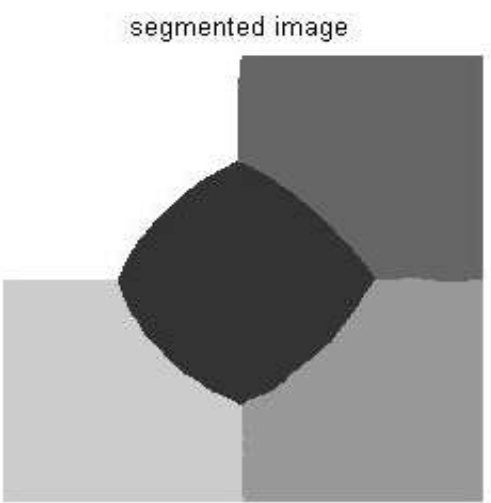

(b)

Figure 4.Segmentation of five texture gray scale image (a) input image(d) segmented image for $\theta=$ $30^{\circ}$ and $N_{c}=204$ pixels

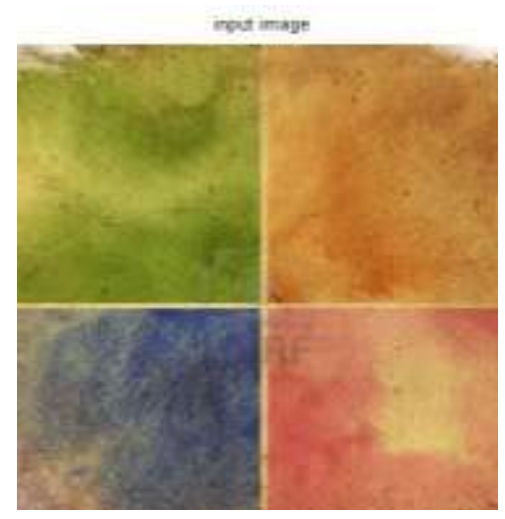

(a)

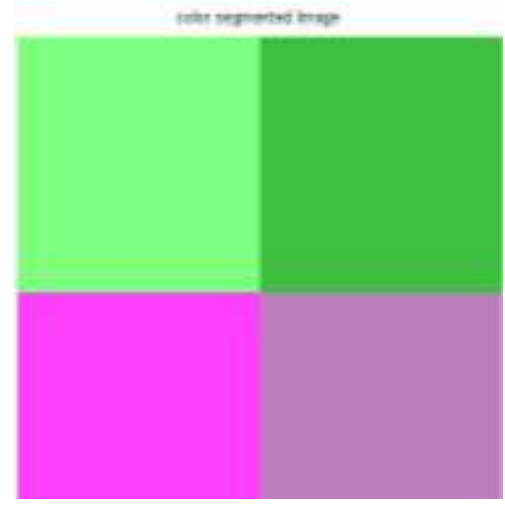

(b)

Figure 5.Segmentation of four texture colour image (a) input image (b) segmented image for $\theta=45^{0}$ and $N_{c}=$ 225 pixels

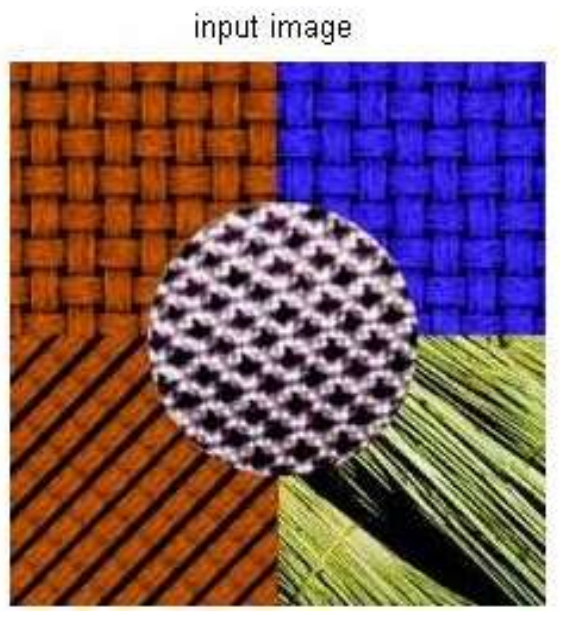

(a)

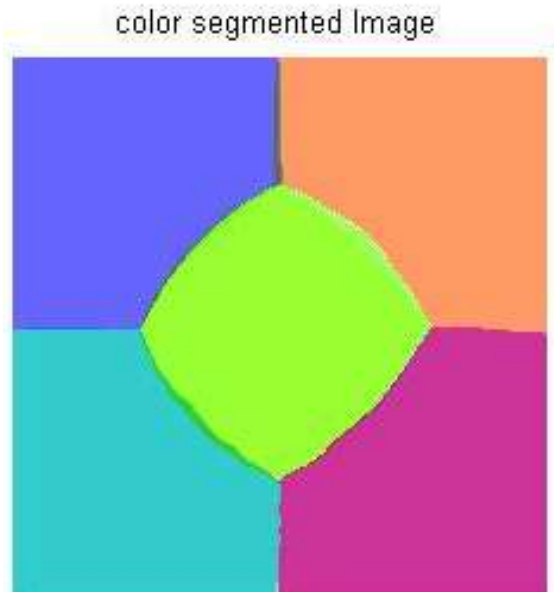

(b)

Figure 6.Segmentation of five texture image (a) input image(d) segmented image for $\theta=30^{0}$ and $N_{c}=225$ pixels

The segmentation four textured and five textured gray scale and colour images were observed here. From the results it is clear that the multichannel Gabor filtering scheme was suitable for the segmentation of multi textured images. And also from the results we observed that the edges of the textures are discriminated clearly. 


\section{Conclusion}

Colour and gray scale texture segmentation is achieved using multichannel Gabor filtering and results are encouraging. First filter bank was designed by considering different frequencies and orientations, then the image is passed through the bank. The image is decomposed into the number of filtered images. Then we extract the features from the filter bank outputs. The final step is clustering the pixels, after clustering each pixel is labelled producing the segmented image.

In the present work, multi channel texture segmentation for colour images has been carried out based on component separate approach. This approach is rather tedious for colour images. Possibilities can be explored to use quaternions, a 4-D extension of complex numbers to represent colour pixels and apply multi channel Gabor filtering for colour texture segmentation.

\section{References}

[1] Todd R. Reed and J. M. H. Du Buf, “A review of recent texture segmentation and feature extraction techniques", Computer Vision, Graphics, and Image Processing, Vol. 57, no.3, pp. 359-372, 1993.

[2] A. K. Jain, Farshid Farrokhnia, “Unsupervised texture segmentation using Gabor filters”, Pattern Recognition”, Vol. 4, no. 12, pp. 1167- 1186, 1991

[3] A. C. Bovik, M. Clark, W. S. Geisler, "Multichannel texture analysis using localized spatial filters", IEEE trans. On pattern analysis and machine intelligence, Vol. 21, no. 1, pp. 55-73, 1990.

[4] J. Malik, P. Perona, "Preattentive texture discrimination with early vision mechanisms", J. Opt. Soc. Am. A, Vol. 7, pp 923-932, 1990 .

[5] M. R. Turner, "Texture discrimination by Gabor functions", "Biological Cybernetics", Vol. 55, pp. 71-82, 1986.

[6] D. Clausi, M. Ed Jernigan, "Designing Gabor filters for optimal texture separability”, PatternRecognition, vol. 33, pp. 1835-1849, 2000 .

[7] K. R. Castleman, "Digital Image Processing,” Prentice Hall, 1996.

[8] M. Clark, A. Bovik, W. Geisler, "Texture segmentation using a class of narrowband filters", IEEE CH2396-0, 1987.

[9] A. K. Jain, F. Farrokhnia, "Unsupervised texture segmentation using Gabor filters", Pattern Recognition, vol. 24, no. 12, pp. $1167-1186,1991$.

[10] P. Brodatz, "Textures: A Photographic Album for Artists and Designers", Dover, New York, 1966.

[11] O. Pichler, A. Teuner, B. Hosticka, "An unsupervised texture segmentation algorithm with feature space reduction and knowledge feedback”, IEEE Trans. Image Proc, vol. 7, no. 1, 1998.

[12] T. P. Weldon, W. E. Higgins, D. F. Dunn, "Gabor filter design for multiple texture segmentation".

[13] Khaled Hammouda, "Texture segmentation using Gabor filters", 2002. 